\title{
Über den Einfluss der Temperatur auf die Ozonbildung
}

\author{
von \\ Alfred Beill. \\ (Mit 1 Tafel.) \\ Aus dem chemischen Laboratorium des Prof. Ad. Lieben an der \\ k. k. Universität in Wien.
}

(Vorgelegt in der Sitzung am 12. Jänner 1893.)

Von den zahlreichen Untersuchungen, welche sich in der Literatur über diesen Gegenstand vorfinden, erwähne ich folgende:

L. Babo ${ }^{1}$ liess Sauerstoff durch sein Ozonisationsrohr strömen, unter Abkühlung, eventuell Erwärmung desselben. Er bestimmte die Ozonmenge in dem über Wasser aufgefangenen Gase mittelst Jodtitration. Es wurden Versuche zwischen $-21^{\circ} \mathrm{C}$. und $+140^{\circ} \mathrm{C}$. gemacht, die Resultate fielen aber ganz unregelmässig aus. Er schliesst daraus, dass zwischen $-19^{\circ} \mathrm{C}$. und $+95^{\circ}$ C. die Differenz der Temperatur so gut wie keinen Einfluss auf die Stärke der Ozonisation ausübt, dass diese aber von $\mathrm{da}$ an allmälig schwächer wird und über $+130^{\circ} \mathrm{C}$. kaum mehr erfolgt.

Hautefuille und Chappuis ${ }^{2}$ benutzten einen Apparat von Berthelot, um eine gegebene Menge Sauerstoff, unter Variiren des Druckes und der Temperatur zu ozonisiren. Bei $760 \mathrm{~mm}$ Druck fanden sie folgendes Gewichtsverhältniss an Ozon:

1 Annalen der Chem. und Pharm., 1863, Supplementband II.

2 Compt. rend, 91 . 


$$
\begin{array}{crr}
\text { Für Temperatur } & -23^{\circ} \ldots \ldots \ldots 0 \cdot 214 \\
\gg & 0^{\circ} \ldots \ldots \ldots .149 \\
\gg & \Rightarrow & +20^{\circ} \ldots \ldots \ldots .0 \cdot 106
\end{array}
$$

Noch eine Arbeit will ich erwähnen, wenn auch dieselbe in keiner directen Beziehung mit der vorliegenden steht. Es sind dies die Untersuchungenvon Th. Andrew s und Tait. ${ }^{1}$ Ich habe einige von den beiden Forschern beobachteten Phänomene bestätigt und mein Apparat war nur eine Modification des inrigen.

Meine ersten Versuche, bei denen ich Sauerstoff durch eine Siemens'sche Röhre streichen liess, das Ozon mittelst arseniger Säure ${ }^{2}$ absorbirte, und mit Jod titrirte, ergaben ganz unregelmässige Resultate. Doch wenn ich die Versuche, die unter sonst gleichen Bedingungen (Temperatur, Stromstärke) gemacht wurden, nach der Schnelligkeit, mit welcher Sauerstoff die Ozonisationsröhre passirte, zusammenstellte, zeigte sich eine

\begin{tabular}{|c|c|c|c|c|}
\hline $\begin{array}{l}12 \text { passirte den Ozonigator in } \\
\text { Minuten } \ldots \ldots \ldots \ldots \ldots \ldots\end{array}$ & $13 i$ & 174 & 192 & 266 \\
\hline $\begin{array}{l}\text { Ozonmenge }\left(\mathrm{O}_{3}\right) \text { in } 12 \text { berechnet } \\
\text { in Grammen } \ldots \ldots \ldots \ldots \ldots\end{array}$ & 0.054 & 0.064 & 0.071 & 0.092 \\
\hline
\end{tabular}
Regelmässigkeit, wie folgende Tabelle beweist:

Beì Temperatur $0^{\circ} \mathrm{C}$.

Die Zeit übt somit einen nicht zu unterschätzenden Einfluss auf die Ozonbildung, ich entschloss mich daher, eine bestimmte Sauerstoffmenge durch eine bestimmte Zeit dem Inductionsstrome auszusetzen.

Mein Apparat (Fig. 1) bestand aus zwei ineinandergeschobenen, oben zusammengelötheten Glasröhren (a), von denen die äussere aussen, die imnere innen mit Stanniol belegt war. Die lichte Weite betrug $1.8 \mathrm{~cm}$ und $0.9 \mathrm{~cm}$, die Dicke des Glases $0.93 \mathrm{~mm}$ und $0.88 \mathrm{~mm}$, die Länge des Ozonigators $9 \mathrm{~cm}$, Volumen $14.94 \mathrm{~cm}^{3}$. An diese Ozonisationsröhre wurde

1 Pogg. Ann., 112 und 152.

2 Thenard., Compt. rend., 1872. 
oben ein dickwandiges, mit einem sehr guten Geissler'schen Hahne (c) versehenes Capillarrohr angelöthet, unten ein langes ebensolches Rohr, welches die Verbindung mit der Messvorrichtung herstellte. Die letztere (b) bestand aus einem in $1 / 100 \mathrm{~cm}^{3}$ getheilten Glasrohr, an welches ein weiteres angelöthet war. Wie aus der Zeichnung ersichtlich, gestattete die Messvorrichtung, das Gas mit Schwefelsäure abzusperren, und durch Zuoder Abfliessenlassen derselben das Gas unter Atmosphärendruck zu beobachten.

Bei $d$ befand sich ein Glasschliff, welcher ermöglichte, das System mit einer Wasserstrahlsaugpumpe in Verbindung $z \mathrm{u}$ setzen. Der Ozonigator (a) befand sich in einem cylindrischen Gefässe (e), welches oben mittelst eines Korkes (l) luftaicht verschlossen war. Das Gefäss gestattete drei Verbindungen $(f, g, h) . i$ ist ein Thermometer, $k$ ein mit Luft gefüllter Mantel, welcher das Gefäss (e) vor äusseren Temperaturveränderungen schützte.

Ich entwickeite Sauerstoff aus Mangansuperoxyd in einem schwerschmelzbaren Glasrohre (in), welches mittelst eines Kautschukstöpsels mit dem Quecksilbermanometer (m) und weiter mit einem mit Glasperlen und Schwefelsäure gefüllten U-Rohr in Verbindung stand.

Das Volum des Ozonigators (a) wurde durch Wägen bestimmt.

Alle Theile des Apparates wurden vorher mit Salpetersäure, Wasser und Alkohol ausgewaschen und getrocknet; das Quecksiber ausgekocht; Mangansuperoxyd mit einer kleinen Quantität chlorsaurem Kali stark erhitzt, ausgewaschen, getrocknet, schwach geglüht und in noch heissem Zustande in das Rohr (n) gebracht. Ich verband das Rohr mit dem Apparate, nachdem ich noch einen losen Glaswollebausch eingeschoben habe. Der Hahn $c$ wurde aufgemacht, bei $d$ das Rohr der Saugpumpe mittelst eines Schliffes angesetzt und das ganze System bis auf wenige Centimeter, unterEwärmung der leichter zugäng. lichen Theile, ausgepumpt. Hierauf schloss ich die Pumpe ab. und erhitzte stark einen kleinen Theil des Mangansuperoxyd. Nachdem sich der ganze Apparat mit Sauerstoff gefült hat, liess ich die Pumpe von neuem wirken und wiederholte die 
Procedur drei- bis viermal. Auf diese Weise erhielt ich den Ozonisator mit vollkommen reinem Sauerstoff gefüllt.

Als Stromquelle dienten drei Bunsenelemente. Die betreffende Anordnung ist aus dem Schema (Fig. 2) ersichtlich. Da die Intensität des Stromes von grossem Einflusse auf die Menge des gebildeten Ozons ist, war es immer mein Streben, mit möglichst gleichmässigen Strömen zu arbeiten. Die gröberen Stromschwankungen wurden durch stärkere oder schwächere Concentration der in den Elementen befindlichen Schwefelsäure ausgeglichen. Bei feineren Schwankungen geschah dies mittelst eines einfachen Rheostaten. Derselbe bestand aus zwei $2.5 \mathrm{~m}$ langen, parallel laufenden Eisendrähten, zwischen welchen ein verschiebbarer Quecksilbercontact hergestellt war. Zur Controle diente ein Ampérometer. Dasselbe war derart eingeschaltet, dass es sich durch Eintauchen des Verbindungsdrahtes in das eine oder andere Quecksilbernäpfchen in oder ausser Function setzen liess. Diese Anordnung ermöglichte, ohne Zeitverlust die eventuellen Stromschwankungen auch während des Ganges der Ozonisation von Zeit zu Zeit zu beobachten.

Der Apparat zur Messung der Funkenlänge (Fig. 3) bestand aus einem Glaskugelrohr von circa $7 \mathrm{~cm}$ Durchmesser, welches mit einem $\mathrm{CaCl}_{2}$-Thurm verbunden war. Bei $a$ wurde ein Pt-Draht eingeschmolzen, der Draht $b$ liess sich in einer Korkführung verschieben. An demselben war eine Marke (c) angebracht, und an einer daneben befindlichen Scala (d) konnte man den Abstand beider Drähte ablesen. Behufs Messung der Funkenlänge wurden die beiden Drähte mit den Polen des Inductionsapparates leitend verbunden, mittelst eines bei $e$ angesetzten Aspirators ein langsamer Luftstrom durchgezogen, die Spitzen soweit einander genähert, bis regelmässige Funken durchzuschlagen begannen. Dieser Abstand wurde an der Scala abgelesen.

Ich liess den Strom immer 30 Minuten durch den Ozonisationsapparat gehen. Zwar beobachtete ich bei einigen speciell zu diesem $Z$ wecke ausgeführten Versuchen, dass die Ozonisation über zwei Stunden dauern kann, doch sind die Mengen des sich bildenden Ozons bereits so klein, dass sie ausser Acht gesetzt werden können, um so mehr, wenn es sich um ver- 
gleichende Bestimmungen, also um relative Mengen handelt. Der weitaus grösste Theil bildet sich in den ersten 10 Minuten, nach 20 Minuten ist gewöhnlich eine Volumverminderung bereits kaum bemerkbar.

Um in verschiedenen Temperaturen arbeiten zu können, wurden die drei Öffnungen $(f, g, h)$ des Cylinders (e) mit entsprechenden Apparaten verbunden.

Für Temperaturen $z$ wischen $0^{\circ} \mathrm{C}$. und $+20^{\circ} \mathrm{C}$. setzte ich bei $f$ den Schlauch einer Körting'schen Wasserstrahl-Saugpumpe an. Durch die Öffnung $h$ trat die mit Schwefelsäure getrocknete Luft ein. Das Gefäss $e$ wurde mit Äther gefüllt, der verdunstete Äther durch frischen ersetzt, mittelst einer bei $g$ angebrachten Verbindung. Sonst war die Öffnung $g$ geschlossen. Zur Regulirung des Luftstromes dienten zwei bei $f$ und $h$ angebrachte Schraubenquetschhähne. Bei dieser Anordnung liess sich die Temperatur mit Leichtigkeit sehr constant halten. Die momentanen Schwankungen betrugen kaum $1^{\circ} \mathrm{C}$.

Für Temperaturen über $+20^{\circ} \mathrm{C}$. wurden Dämpfe schlechter Leiter: Äther, Chloroform, Alkohol, Toluol, Xylol, Anilin benützt. $\mathrm{Zu}$ diesem $\mathrm{Zwecke}$ wurden die erwähnten Flüssigkeiten in einem circa $200 \mathrm{~cm}^{3}$ fassenden Kolben bis zum Sieden erhitzt, und derKolben mit dem Gefässe derart verbunden, dass der Dampf bei $g$ eintrat, und die condensirte Flüssigkeit durch $h$ in den Kolben zurückkehrte. Bei $f$ setzte ich einen Rückflusskühler an. Die beobachteten Temperaturen entsprechen nicht genau den Siedepunkten der betreffenden Stoffe, weil die letzteren nicht rein waren, doch wurden constante Temperaturen erhalten.

Für Bestimmungen bei Temperatur $-20^{\circ} \mathrm{C}$. und $-45^{\circ} \mathrm{C}$. wurde der Kork ( $l$ ) entfernt, die Öffnungen $h$ und $g$ geschlossen, das Gefäss (e) mit Äther gefüllt und durch Zugabe fester Kohlensäure, die Temperatur erniedrigt. Durch Eintragen von ganz kleinen Stücken $\mathrm{CO}_{2}$ liess sich die Temperatur bis.auf wenige Grade constant halten.

Die Temperatur $-73^{\circ} \mathrm{C}$. erhielt ich mittelst eines $\mathrm{CO}_{2}$ Breies. $Z u$ diesem Behufe wurden die beiden Gefässe $e$ und $k$ entfernt, durch entsprechende Biegung der Glasröhren der Ozonigator in horizontale Lage gebracht und in ein aus starker Pappe angefertigtes, mit einem dicken Tuchüberzug versehenes 
Gefäss gelegt. Ich füllte das Pappegefäss mit fester Kohlensäure und befeuchtete diese mit einigen Tropfen Äther.

Die Temperaturen über $-20^{\circ} \mathrm{C}$. wurden mit einem Quecksilberthermometer unter $-20^{\circ}$ C. mit einem Schwefelkohlenstoffthermometer gemessen. Die Scala des letzteren wurde mittelst Bestimmung des Gefrierpunktes des Wassers und des Quecksilbers corrigirt.

In der unten angegebenen Versuchsreihe ist der Procentgehalt an Ozon verhältnissmässig klein (Maximum $10.4 \%$ bei $-73^{\circ}$ C.). Der Grund davon ist in den starken Glaswänden meiner Ozonisationsröhre und in dem schwachen Inductionsstrome zu suchen. Doch lagen besondere Gründe vor, dem absoluten Procentgehalte nur eine untergeordnete Beachtung zu schenken: Das Wichtigste war eine grössere Anzahl der Bestimmungen in ein und demselben Apparate unter möglichst denselben Umständen auszuführen. Bei Anwendung starker Ströme, eventuell schwacher Glaswände des Ozonigators, kam es zu oft vor, dass ein zufälligerweise stärkerer Funke - bei Rumkorff'schen Inductionsapparaten mit Stanniolcondensatoren kommen solche oft vor - den Apparat zerschlug. Mit zunehmender Stromstärke oder, was auf dasselbe herauskommt, mit abnehmender Glasdicke des Ozonigators, wächst die Ozonmenge rapid. Spuren von Verunreinigungen, insofern sie den Leitungswiderstand des Sauerstoffs vermindern, wirken ebenfalls fördernd auf die Ozonmenge. Hau tefuille und Chap puis ${ }^{1}$ beobachteten, dass eine Beimischung von Stickstoff oder Wasserstoff auf die Ozonbildung günstig wirkt, wie auch, dass - bei gleicher Temperatur und Druck - die Menge des gebildeten Ozons beträchtlich grösser ist bei Gegenwart von Wasserstoff als bei Gegenwart von Stickstoff.

Der Umstand, dass reiner Sauerstoff mit abnehmender Temperatur immer schlechterer Leiter wird, wirkt der Zunahme des Ozongehaltes entgegen. Meine Versuche fingen schon etwas über $0^{\circ} \mathrm{C}$. an, unregelmässige Resultate zu ergeben. Um wieder unter einander stimmende Resultate zu erhalten, entschloss ich mich, das mit Schwefelsäure gefüllte U-Rohr, durch ein Chlor-

1 Compt. rend., 91, p. 762 . 
calciumrohr zu ersetzen, bei welcher Zusammenstellung der Sauerstoff eine Spur Feuchtigkeit enthält. Die entsprechenden Versuche ergaben eine Linie von derselben Neigung wie bei reinem Sauerstoffe, nur wurde der Procentgehalt an Ozon etwas erhöht.

Die Berechnung geschah auf Grund der Volumverminderung. Die Grösse der Contraction wurde mit 2 multiplicirt und daraus der Procentgehalt berechnet. Die angegebenen Zahlen sind also Volumprocente. Da bei Temperaturen unter $0^{\circ} \mathrm{C}$. der Vergleich der Volumina vor und nach dem Versuche, bei genau derselben Temperatur umständlich war, berechnete ich die Ozonmenge mittelst Titration. $\mathrm{Zu}$ diesem Behufe wurde bei $d$ (Fig. 1) ein eingeschliffenes Glasrohr (Fig. 4) angesetzt, und mit einigen Tropfen $\mathrm{H}_{2} \mathrm{SO}_{4}$ gedichtet. In das Rohr wurde reine Glaswolle gebracht und diese mit $1 \mathrm{~cm}^{3}$ titrirter Arseniksäurelösung befeuchtet. Dann erhitzte ich das Mangansuperoxyd (Fig. 1 ) mässig, öffnete den Hahn $c$ und spülte mit einem sehr langsamen Strome Sauerstoffs das Ozon aus dem Ozonigator aus. Auf diese Weise gelang es, das Ozon vollständig zu absorbiren: das austretende Gas bläute das JK-Papier nicht und war geruchlos. Die überschüssige arsenige Säure titrirte ich, nach Mohr, mit Jod.

Alle hier angegebenen Bestimmungen wurden unter folgenden Umständen gemacht:

3 Bunsen'sche Elemente, Stromstärke $2 \cdot 6$ Ampère, Funkenlänge $22 \cdot 5 \mathrm{~mm}$, Dauer der Ozonisation 30 Minuten.

Ich erhielt dabei folgende Zahien:

Reiner Sauerstoff, mit Schwefelsäure getrocknet.

\begin{tabular}{|c|c|c|c|c|}
\hline \multirow{2}{*}{ Temperatur } & \multicolumn{2}{|c|}{ Niveau } & $\begin{array}{c}\text { Contrac- } \\
\text { tion }\end{array}$ & $\mathrm{O}_{3} \%$ \\
\cline { 2 - 5 } & Anfang & Ende & \\
\hline $0^{\circ}$ & $2 \cdot 17$ & 1.815 & 0.355 & 4.7 \\
0 & 2.01 & 1.59 & 0.42 & 5.6 \\
+10 & 1.59 & 1.21 & 0.38 & 5.0 \\
\hline
\end{tabular}




\begin{tabular}{|c|c|c|c|c|}
\hline \multirow{2}{*}{ Temperatur } & \multicolumn{2}{|c|}{ Niveau } & \multirow{2}{*}{$\begin{array}{l}\text { Contrac- } \\
\text { tion }\end{array}$} & \multirow{2}{*}{$\mathrm{O}_{3} \%$} \\
\hline & Anfang & Ende & & \\
\hline$+25^{\circ}$ & $2 \cdot 24$ & $1 \cdot 98$ & 0.26 & $3 \cdot 4$ \\
\hline+25 & $2 \cdot 15$ & $1 \cdot 82$ & 033 & $4 \cdot 4$ \\
\hline+35 & $i \cdot 73$ & $1 \cdot 42$ & 0.31 & $4 \cdot 1$ \\
\hline+35 & $1 \cdot 775$ & $1 \cdot 55$ & 0.225 & $3 \cdot 0$ \\
\hline+59 & $2 \cdot 955$ & $2 \cdot 73$ & 0.225 & $3 \cdot 0$ \\
\hline+59 & $2 \cdot 422$ & $2 \cdot 26$ & 0.162 & $2 \cdot 1$ \\
\hline+78 & $0 \cdot 80$ & 0705 & 0.095 & $1 \cdot 3$ \\
\hline+78 & $2 \cdot 023$ & $1 \cdot 895$ & 0.128 & $1 \cdot 6$ \\
\hline+109 & $1 \cdot 755$ & $1 \cdot 692$ & 0.063 & 0.8 \\
\hline+109 & $2 \cdot 475$ & $2 \cdot 43$ & 0.045 & $0 \cdot 6$ \\
\hline+132 & $2 \cdot 13$ & $2 \cdot 105$ & 0.025 & $0 \cdot 3$ \\
\hline+170 & mitte & J $\mathrm{K}-\mathrm{P}$ & er nachwe & sbar. \\
\hline
\end{tabular}

Bei chlorcalcium-trockenem Sauerstoffe erhielt ich folgende Ergebnisse:

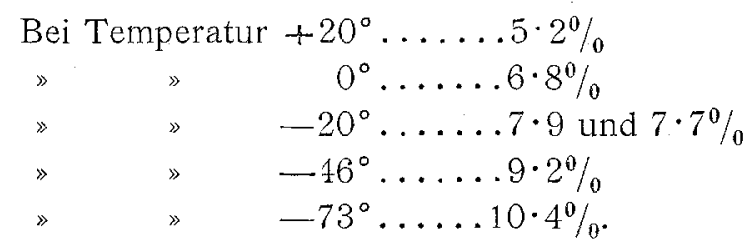

Die Ozonmenge wurde bei $-20^{\circ},-46^{\circ}$ und $-73^{\circ}$ mittelst oben erwähnter Titration bestimmt.

Im Coordinatensystem (Fig.6) geordnet, bilden die relativen Ozonmengen eine fast gerade Linie. Die obere Linie - zwischen $+20^{\circ}$ und $-73^{\circ}-$ wurde aus $\mathrm{CaCl}_{2}$-trockenem, die untere - zwischen $0^{\circ}$ und $+170^{\circ}$ - aus $\mathrm{SO}_{4} \mathrm{H}_{2}$-trockenem Sauerstoffe erhalten. Mit abnehmender Temperatur wächst der Ozongehalt in reinem Sauerstoff ständig, aber sehr langsam. Ich vermuthe, dass die mit der fallenden Temperatur abnehmende Leitungsfähigkeit des reinen Sauerstoffes der schnellen Zunahme an Ozongehalt entgegenwirkt. 
Ich will noch einen interessanten Zustand des Sauerstoffes erwähnen, den ich gelegentlich beobachtet habe. Zu meinen Vorversuchen benützte ich unter anderem ein Ozonisationsrohr (Fig. 5), welches, nachdem es mit möglichst reinem Sauerstoff gefüllt war, einerseits zugeschmoizen wurde. Ich beabsichtigte, nach einer jeden Bestimmung das Ozon mittelst glühenden Platindrahtes zu zerstören, ${ }^{1}$ um eine ganze Versuchsreihe auszuführen, ohne den Apparat mit frischem Sauerstoff füllen zu müssen. Die Resultate waren ganz zufriedenstellend, doch nach einer längeren Reihe von Bestimmungen bemerkte ich sehr grosse Unregelmässigkeiten. Ich fand, dass sich der Sauerstoff, wenn er knapp nach dem Glühen des Pt-Drahtes der Wirkung des Inductionsstromes unterworfen wurde, sehr oft nicht ozonisiren liess. Es trat eine rapide Volumvergrösserung ein, die so lange unverändert dauerte, bis der Strom geöffnet wurde. War dies geschehen, so verminderte sich das Volum langsam, bis es das ursprüngliche Niveau erreichte. Je stärker und länger der Pt-Draht geglüht wurde, desto leichter trat dieser Zustand ein. Dieser Zustand war labil: er dauerte circa eine Stunde, manchmal länger. Nach Verlauf dieser Zeit liess sich der Sauerstoff von neuem ozonisiren, wie zuvor.

Einen ähnlichen Zustand beobachteten auch Andrews und Tait. ${ }^{2}$ Nach ihnen wird dieser erzeugt, wenn man einige Minuten lang einen Strom starker, elektrischer Funken durch Sauerstoff leitet, der eine Spur von Stickstoff enthält. Sie geben an, dass durch diese Behandlung der Sauerstoff unfähig wird sich unter der Wirkung der stillen Entladung zusammenzuziehen und in Ozon zu verwandeln; er erlangt nur durch Erwärmung oder durch ein mehrstündiges Stehenlassen seine gewöhnliche Beschaffenheit. Wenn der Stickstoff nicht mehr als $1 / 500$ des ganzen Volumens betrug, konnten sie diesen Zustand nicht mehr als zwei- oder dreimal hervorbringen. Sie erklären ihn durch die Gegenwart einer Spur Untersalpetersäure, die durch die elektrischen Funken erzeugt wird.

Mein Apparat war mit elektrolytischem, möglichst reinem Sauerstoff gefü11t. Zur Elektrolyse benützte ich concentrirte

1 Compt. rend., 57 , Soret.

a A. a. 0 . 
ausgekochte Kupfersulfatlösung. Der fertig zusammengestellte Apparat wurde unter Erwärmung der leichter zugänglichen Theile ausgepumpt und der Strom von drei starken Bunsenelementen auf 24 Stunden geschlossen. Hierauf wurde der Ozonigator einerseits zugeschmolzen, anderseits mit $\mathrm{H}_{2} \mathrm{SO}_{4}$ abgesperrt. Eine nachherige Veränderung des abgesperrten Gases ist daher ausgeschlossen.

Dennoch beobachtete ich nach einer längeren Versuchsreihe die oben angegebene Veränderung des Sauerstoffzustandes. Wenn diese, auch in meinem Falle in der Wirkung einer Spur von Stickstoff ihren Grund hätte, so bliebe es unaufgeklärt, warum dieser Zustand erst nach einer längeren Versuchsreihe zum Vorschein kam." Es steht der Gedanke am nächsten, dass hier die Ursache in einer Veränderung des Platindrahtes durch starkes Glühen zu suchen ist. Man kann an fein zerstäubtes Platin oder an dessen unbeständige Sauerstoffverbindungen denken, welche die Ozonbildung hemmen.

Zum Schlusse sei es mir gestattet, Herrn Professor Lieben für die freundliche Unterstützung $z u$ danken, welche er mir bei der Ausführung dieser Arbeit gewährt hat. 
A. Eeill: Temperatur und Ozonbildures
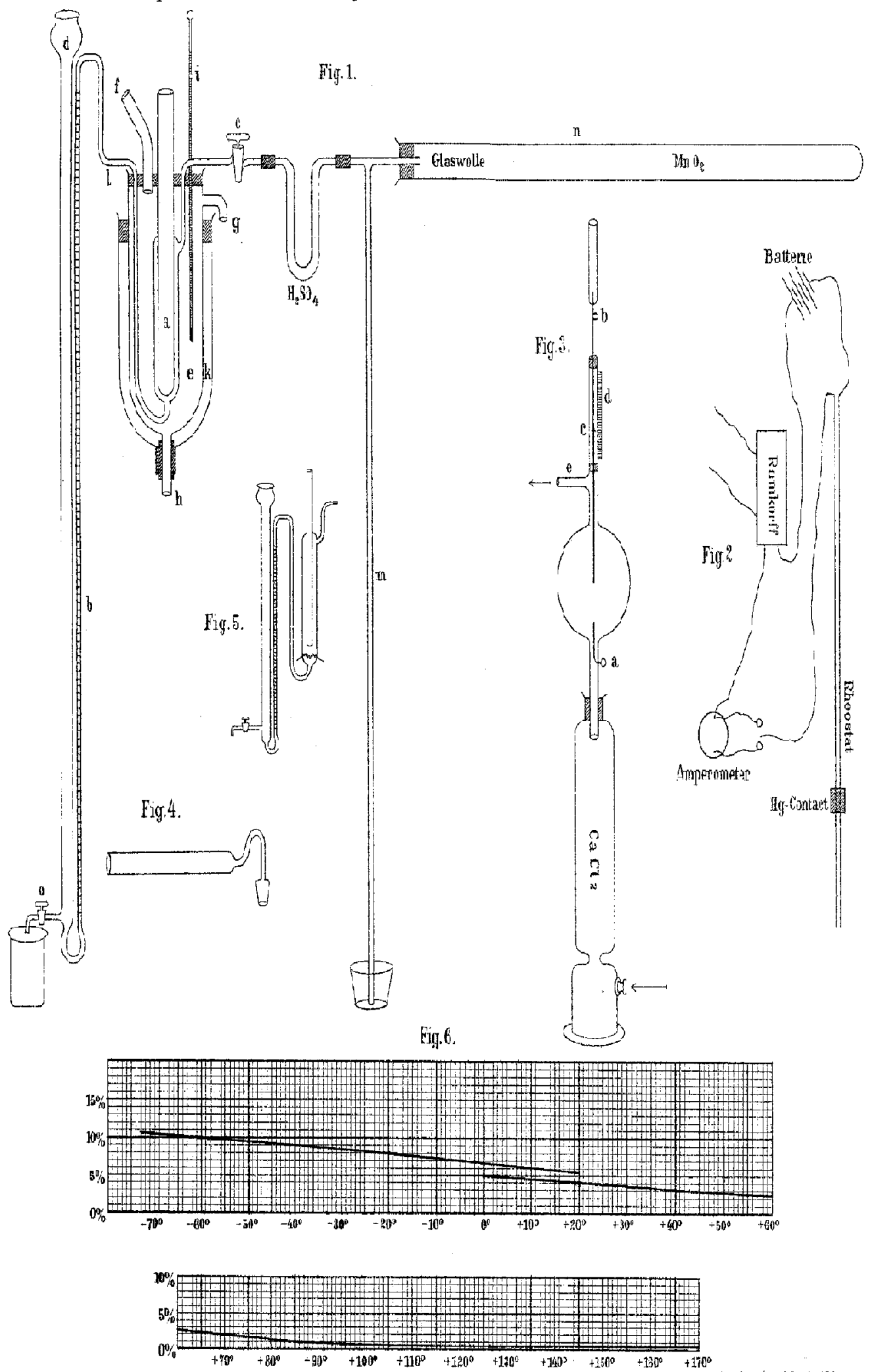